OPEN ACCESS

Edited by:

Yujie Chen,

Army Medical University, China

Reviewed by:

Xiaofei $\mathrm{Hu}$,

Army Medical University, China

Sayona John,

Rush University, United States

Jiang Wu,

Soochow University, China

*Correspondence: Feng Gao

2202012@zju.edu.cn

Lu-sha Tong

2310040@zju.edu.cn

${ }^{\dagger}$ These authors have contributed equally to this work

Specialty section: This article was submitted to

Multiple Sclerosis and

Neuroimmunology,

a section of the journal

Frontiers in Immunology

Received: 02 June 2021 Accepted: 14 July 2021 Published: 29 July 2021

Citation:

Ye X-h, Zhang J-I, Jin Y-j, Shen D, Hao X-d, Li J-w, Zhong J-w, Jin L-h,

Tong L-S and Gao F (2021)

Association Between Insulin

Resistance and Remote DiffusionWeighted Imaging Lesions in Primary Intracerebral Hemorrhage.

Front. Immunol. 12:719462. doi: 10.3389/fimmu.2021.719462

\section{Association Between Insulin Resistance and Remote Diffusion- Weighted Imaging Lesions in Primary Intracerebral Hemorrhage}

\author{
Xiang-hua Ye ${ }^{1}$, Jian-li Zhang ${ }^{1,2}$, Yu-jia Jin ${ }^{1}$, Dan Shen ${ }^{1}$, Xiao-di Hao ${ }^{1,3}$, \\ Jia-wen $\mathrm{Li}^{1}$, Jia-wei Zhong ${ }^{1}$, Lu-hang Jin ${ }^{1}$, Lu-sha Tong ${ }^{1 * t}$ and Feng Gao ${ }^{1 * t}$ \\ ${ }^{1}$ Department of Neurology, The Second Affiliated Hospital, Zhejiang University School of Medicine, Hangzhou, China, \\ 2 Department of Neurology, Lishui Hospital, Zhejiang University School of Medicine, Lishui, China, ${ }^{3}$ Department of Neurology, \\ Henan Provincial People's Hospital, People's Hospital of Zhengzhou University, Zhengzhou, China
}

Background: Abnormal glucose metabolism was shown to be associated with the occurrence of remote diffusion-weighted imaging lesions (R-DWILs) after primary intracerebral hemorrhage $(\mathrm{ICH})$ onset. Insulin resistance is a metabolic disorder that was regarded as an indicator of chronic systemic inflammation. In this study, we aimed to determine the effect of insulin resistance on the occurrence of R-DWILs in ICH.

Methods: Patients with primary $\mathrm{ICH}$ within 14 days after onset were prospectively enrolled from November 2017 to October 2019. R-DWILs was defined as remote focal hyperintensity from the hematoma in DWI, with corresponding hypointensity in apparent diffusion coefficient. The homeostasis model assessment of insulin resistance (HOMA-IR) was used for insulin resistance estimation and calculated as fasting insulin $(\mu \mathrm{U} / \mathrm{ml}) \times$ fasting glucose $(\mathrm{mmol} / \mathrm{L}) / 22.5$. Patients in our cohort were divided into four groups according to HOMA-IR index quartiles. Logistic regression analysis and smoothing plots were used to evaluate the association of HOMA-IR with R-DWIL occurrence. Sensitivity analysis was performed in non-diabetic patients, non-obese patients, hypertensive $\mathrm{ICH}$ patients, and patients 60 years and older separately. The association between HOMA-IR and systemic inflammatory immune indices neutrophil to lymphocyte ratio (NLR) and monocyte to lymphocyte ratio (MLR) was examined with multiple linear regression analysis.

Results: Among the 345 patients, 54 (15.7\%) had R-DWILs. Both the third and fourth quartiles of HOMA-IR index were robustly associated with an increased risk of R-DWIL occurrence (adjusted OR 3.58, 95\% Cl 1.33-9.65; adjusted OR 3.91, 95\%Cl 1.47-10.41) when compared with the first quartile. The association was consistent in non-diabetic, non-obese, hypertensive ICH patients, as well as in patients 60 years and older. Furthermore, both NLR and MLR were independently associated with HOMA-IR. 


\begin{abstract}
Conclusions: Our study suggested that insulin resistance evaluated with HOMA-IR index was independently associated with the presence of R-DWILs in patients with acute and subacute primary $\mathrm{ICH}$. It may provide new insights into the metabolism-related brain injury after $\mathrm{ICH}$ ictus.
\end{abstract}

Keywords: intracerebral hemorrhage, insulin resistance, metabolism, remote diffusion-weighted imaging lesions, inflammation

\section{INTRODUCTION}

Intracerebral hemorrhage (ICH) is one of the most prevalent subtypes of stroke and remains a significant cause of morbidity and mortality worldwide. Despite much efforts made in the past decades, no substantial improvement has emerged to improve functional outcomes after ICH (1). Nevertheless, glucose management has long been proposed to prevent unfavorable outcomes in both nondiabetic and diabetic patients with ICH, although no optimal criteria have been established (2). A better understanding of the impact of glucose metabolism on ICH is in urgent need. We have kept on focusing on the associations between glucose-related indices and the occurrence of remote diffusion-weighted imaging (DWI) lesions (R-DWILs) in brain MRI, which was characterized as hyperintensity lesions in DWI with corresponding hypointensity lesions in apparent diffusion coefficient (ADC), remote from hematoma location topographically $(3,4)$. Although commonly subclinical in the acute stage of ICH, R-DWILs portend worse outcomes as reported by previous studies (5-9). The etiology of R-DWILs is not fully elucidated. It is supposed to be a secondary injury of hemorrhage and probably related to cerebral local inflammation and blood brain barrier (BBB) disruption (3).

Our prior study discovered that increased admission fasting glucose, but not diabetes history, was positively associated with R-DWIL occurrence (3). It may be partially because of the prevalence of abnormal glucose metabolism in patients without diabetes but presenting with hyperglycemia under critical illness (4). Insulin resistance, a systemic metabolic disorder, and the hallmark of type 2 diabetes, is common in patients without diabetes but bearing vascular disease. Impaired insulin sensitivity has emerged as an important contributor to the development and unfavorable prognosis of ischemic stroke (10-15). The possible mechanisms may be attributed to hyperglycemia, hyperinsulinemia, dyslipidemia, hypertension, abnormal fibrinolysis, endothelia dysfunction, systemic inflammation, and atherogenesis (10). Moreover, insulin resistance is interconnected with neuroinflammation and is considered to be one of the major culprits in neurodegenerative process. Also, insulin resistance provides pro-inflammatory effects with profound consequences on the BBB (16-18). Therefore, we hypothesized that insulin resistance is associated with $\mathrm{R}$ DWILs formation in ICH patients.

The homeostasis model assessment of insulin resistance (HOMA-IR) index is a well-established index and has been widely used to assess insulin resistance in clinical studies. In this study, we aim to clarify the hypothesis that insulin resistance measured by HOMA-IR index is associated with R-DWIL occurrence in acute and subacute stage of primary $\mathrm{ICH}$.

\section{MATERIALS AND METHODS}

\section{Study Cohort and Participants}

We prospectively enrolled patients with ICH within 14 days from onset, who were admitted to our hospital from November 2017 to October 2019. ICH was diagnosed based on the American Heart Association/American Stroke Association guideline (2) and confirmed by brain computed tomography (CT).

Primary ICH patients were included in this study when brain MRI was operated within 28 days after onset with standardized protocol and reliable imaging data. Potential patients were excluded from the study if they presented solely intraventricular hemorrhage (IVH) or with secondary causes of $\mathrm{ICH}$, such as arteriovenous malformation, cavernous hemangioma, Moyamoya disease, aneurysm, neoplasm, hemorrhagic conversion of ischemic infarction, or cerebral venous thrombosis. Patients with a recent head trauma were ineligible for our cohort. Those who were deficient in fasting insulin data or being treated with insulin before or during hospitalization were also ruled out (Figure 1 in detail).

\section{Ethics Approval Statement}

The work was approved by the institutional Human Research Ethics Committee of the Second Affiliated Hospital of Zhejiang University. Informed consents were waived due to the observational characteristic of this study, and the data are anonymous.

\section{Data Collection}

Baseline demographic and clinical data, medical histories, vascular risk factors and medical therapies were recorded within $24 \mathrm{~h}$ after admission by trained neurologists. Demographic and clinical information included age, gender, height, weight, body mass index [BMI, calculated as the measured weight $(\mathrm{kg})$ divided by the square of the measured height $\left(\mathrm{m}^{2}\right)$ ], time from symptom onset to hospitalization, time to take the MRI, and collect blood samples. Medical histories and vascular risk factors included history of hypertension, diabetes mellitus, atrial fibrillation (AF), $\mathrm{ICH}$, cerebral infarction (CI)/transient ischemia attack (TIA), smoking, and drinking status. Medical treatments consisted of usage of antiplatelet drug, anticoagulant drug, antihypertensive drug, hypoglycemic agent, and statin before admission. The status of each patient's neurological deficit was evaluated via the 
681 patients with spontaneous ICH registered from November 2017 to October 2019

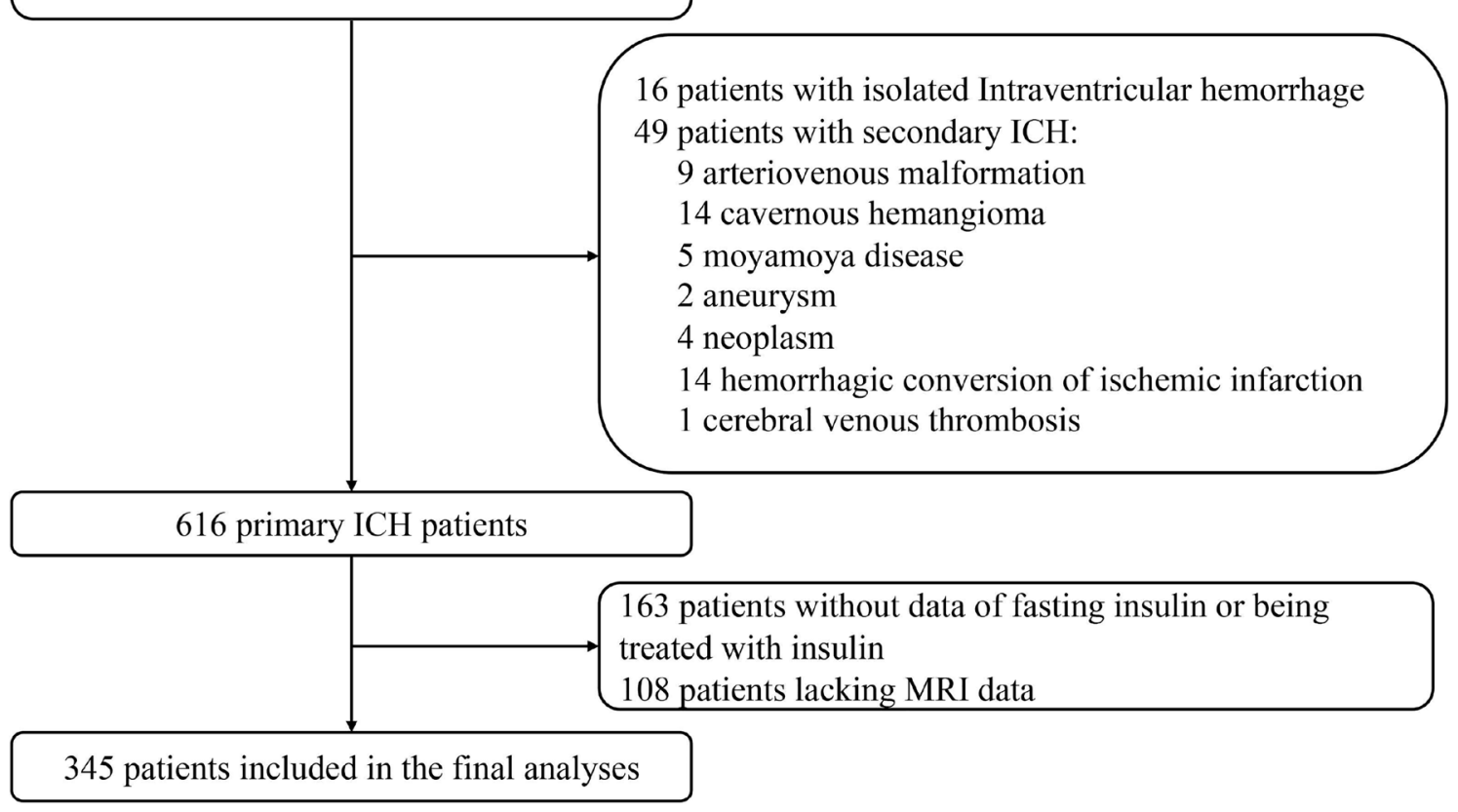

FIGURE 1 | Flow chart of patient enrollment. ICH indicates intracerebral hemorrhage; MRI, magnetic resonance imaging.

National Institutes of Health Stroke Scale score (NIHSS) by neurologists certified for assessing the scale.

The etiologies of ICH were categorized as hypertensive angiopathy (HA), cerebral amyloid angiopathy (CAA), and anticoagulation-related or undetermined cause. Two experienced neurologists (XHY and JLZ) determined the most likely etiology for the qualifying ICH based on available clinical data and neuroimaging, and reached consensus with a Kappa value of 0.98. The CAA-related $\mathrm{ICH}$ diagnosis was made according to the Boston criteria (19).

Blood samples were obtained in the next morning after admission with overnight fasting (at least $8 \mathrm{~h}$ ), and routine laboratory examinations [absolute neutrophil count (ANC), absolute lymphocyte count (ALC), absolute monocyte count (AMC), total cholesterol (TC), low density lipoprotein cholesterol (LDL-C), creatinine, glucose, and insulin] were included. The estimated glomerular filtration rate (eGFR) was calculated as the following Chronic Kidney Disease Epidemiology Collaboration (CKD-EPI) equation for the Asian population: $\mathrm{eGFR}=141 \times \min ($ serum creatinine $/ \kappa, 1) \alpha \times \max$ (serum creatinine $/ \kappa, 1)-1.209 \times 0.993$ age $\times 1.018$ [if female], where $\kappa$ was 0.7 for females and 0.9 for males, $\alpha$ was -0.329 for females and -0.411 for males, min was the minimum of $\mathrm{SCr} / \kappa$ or 1 , and max indicated the maximum of SCr/ $\mathrm{\kappa}$ or $1(20)$.

\section{Measurement of Insulin Resistance}

Blood was collected at 2 days (IQR, 2-4 days) after ICH ictus. The blood levels of fasting glucose and insulin were measured using the hexokinase method and chemiluminescence immunoassay, respectively. Insulin resistance was estimated by the homeostasis model assessment of insulin resistance (HOMA-IR) with the following formula: fasting insulin $(\mu \mathrm{U} / \mathrm{ml}) \times$ fasting glucose $(\mathrm{mmol} / \mathrm{L}) / 22.5$ (21). There was no clear definition of insulin resistance based on HOMA-IR index among different populations. The higher quartiles of HOMA-IR indicated more severe insulin resistance. Furtherly, a HOMA-IR score of more than 3.0 was used for diagnosing insulin resistance in non-diabetic patients in our study, as previously reported in IRIS Trial (22).

\section{Measurement of Systemic Inflammatory Immune Status}

Neutrophil to lymphocyte ratio (NLR) and monocyte to lymphocyte ratio (MLR) are two widely used indicators for evaluating systemic inflammatory immune status. NLR was calculated as ANC divided by ALC. MLR was calculated as AMC divided by ALC (23).

\section{Neuroimaging Protocol}

CT scan was performed on admission and in subsequent review if necessarily, using multidetector row scanners [Optima CT540, General Electric (GE) Healthcare, Connecticut, America; or SOMATOM Sensation 16, Siemens, German] with followed parameters: slice thickness, $5 \mathrm{~mm} ; 120 \mathrm{Kv}$, and 100 to 300 mAs. MRI within 28 days after ICH onset was performed using 1.5-Telsa (T) (Sonata, Siemens, German) or 3.0-T scanner (Signa HDxt; GE Healthcare, Hartford, CT) with 
sequences comprised of axial T1-weighted, T2-weighted, T2 fluid attenuated inversion recovery (FLAIR), DWI [parameters on 1.5-T scanner: repetition time (TR) 3,100 ms, echo time (TE) 84 $\mathrm{ms}, \mathrm{b}=0 / 1,000 \mathrm{~s} / \mathrm{mm}^{2}, 6-\mathrm{mm}$ slice thickness, $0.5-\mathrm{mm}$ gap, field of view (FOV) $230 \times 230 \mathrm{~mm} / 3.0$-T scanner: TR 5,200 ms, TE 75 $\mathrm{ms}, \mathrm{b}=0 / 1,000 \mathrm{~s} / \mathrm{mm}^{2}, 6-\mathrm{mm}$ slice thickness, $0.5-\mathrm{mm}$ gap, FOV $240 \times 240 \mathrm{~mm}$ ], apparent diffusion coefficient (ADC), and T2 star weighted angiography (SWAN).

\section{Assessment of Hematoma}

The size and localization of hematoma were evaluated from initial head CT scan. Hematoma volume was calculated using the ABC/2 method (24). Hematoma location was classified into four types and described as lobar (including frontal, temporal, parietal, occipital, and insular lobe), deep (including basal ganglia and thalamus), infratentorial (brainstem or cerebellum), and mixed (two or more locations above involved). The ventricles and subarachnoid extension were also recorded if present. The collection of hematoma features was done by one certified neurologist (JWZ).

\section{Assessment of R-DWILs}

As reported previously $(3,4,25)$, R-DWILs was defined as a hyperintensity lesion in DWI, with corresponding hypointensity in ADC map, measuring less than $20 \mathrm{~mm}$ in diameter (Figure 2). Restricted diffusion within or adjacent to the hematoma (< $20 \mathrm{~mm}$ ) was not included in our study. DWI sequence and ADC map were read coherently to identify the R-DWILs. The data of R-DWILs was read independently by two experienced and trained readers (J.W.L and X.D.H) blinded to clinical and laboratory data. The inter-rater Cohen-weighted Kappa was 0.96 for the presence of R-DWILs.

\section{Assessment of Total Cerebral Small Vessel Diseases (cSVD) Burden}

The assessment of cSVD burden was conducted as previously published $(4,26)$. Briefly, four MRI markers, including cerebral microbleeds (CMBs), white matter hyperintensities (WMHs), lacunes, and enlarged perivascular spaces (EPVS), was applied to measure cSVD severity. CMBs were defined as rounded or circular foci with low signal intensity on SWAN sequence with a diameter of 2 to $10 \mathrm{~mm}$ (27). WMHs were defined as signal abnormality in white matter with hypointensity in T1-weighted, and hyperintensity in T2-weighted and FLAIR sequences, rating visually on axial FLAIR images according to the four-point Fazekas scale (28). Periventricular white matter hyperintensity (PV-WMH) and deep white matter hyperintensity (D-WMH) were assessed, respectively;
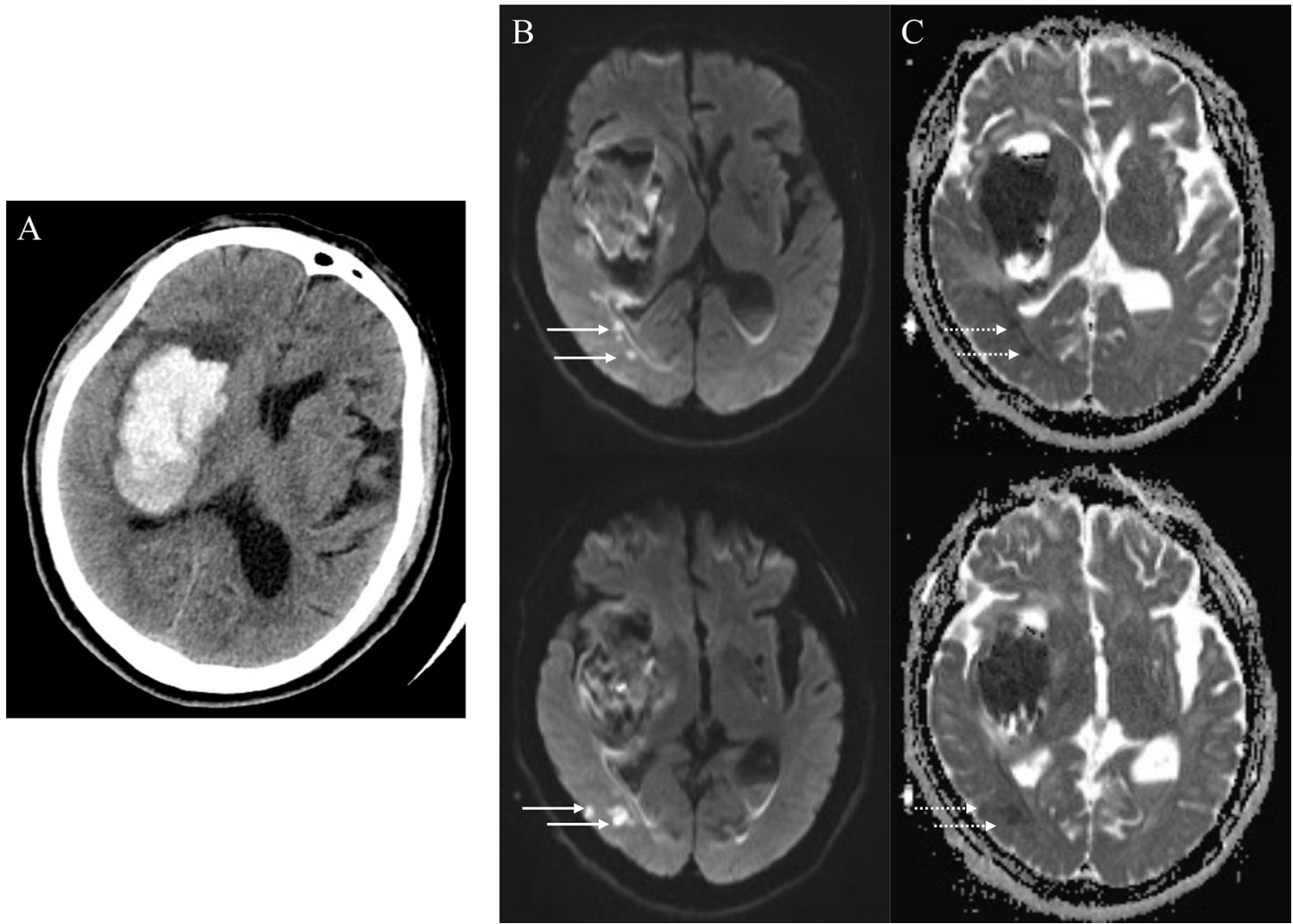

FIGURE 2 | Positive remote diffusion-weighted imaging lesions in a 65-year old man with right basal ganglia hemorrhage (A). Diffusion-weighted imaging shows hyperintensity lesions (B, solid arrowheads) in ipsilateral occipito-temporal lobe, with corresponding hypointensity in apparent diffusion coefficient map (C, dotted arrowheads) at the 8th day after $\mathrm{ICH}$ ictus. 
the overall degree of WMH was calculated as the total of PV-WMH and D-WMH scores. EPVS were defined as $\leq 2 \mathrm{~mm}$ round or linear CSF isointense lesions along the course of penetrating arteries. They were distinguished from lacunes by the size difference (lacunes $>2$ and $\leq 15 \mathrm{~mm}$ ) and surrounding rim of FLAIR hyperintensity (29, 30). EPVS were counted on the brain slice showing the greatest extent of EPVS in the basal ganglia only. The evaluation of EPVS, lacunes, WMHs, and CMBs was performed by one experienced physician (XHY). Total cSVD burden was awarded as follows: one point was scored if there were presence of lacunes, $>20$ BG-EPVS, 1$4 \mathrm{CMBs}$, or three- to four- degrees WMHs, separately; two points was given if with $\geq 5 \mathrm{CMBs}$ or five- to six-degree WMHs, respectively. The range of total cSVD score was therefore 0 to 6 (31).

\section{Statistical Analysis}

Distributions of continuous variables were assessed for normality using the Shapiro-Wilk test. Continuous variables were expressed as median [interquartile range (IQR)] or mean [standard deviation (SD)] for non-normal or normal distributed variables, respectively. Categorical variables were presented as number (percentage). The index of HOMA-IR was classified into four subgroups according to the quartiles. The characteristics of patients were compared using ANOVA or the Kruskal-Wallis test for continuous variables and chi-squared test for categorical variables, among quartiles of HOMA-IR. When comparing the differences between patients with or without R-DWILs, $t$ test, or Mann-Whitney $U$ test was conducted for continuous variables and chi-squared test for categorical variables. Logistic regression modeling was used to evaluate whether HOMA-IR independently predicted the occurrence of R-DWILs after adjusting for potential confounders (age, gender, BMI, NIHSS, history of hypertension and diabetes mellitus, ventricle extension, total cSVD score, and antiplatelet use). Baseline variables, such as age, gender, BMI, history of hypertension, and diabetes mellitus, were assumed clinically relevant to the outcomes of interest, whereas other confounders were put into further analysis if their influence in effect were estimated more than $10 \%$. The relationship between HOMA-IR and R-DWIL occurrence was also explored using smoothing plots. In the sensitivity analysis, we conducted analyses in non-diabetic, non-obese (BMI $<25 \mathrm{~kg} / \mathrm{m}^{2}$, a threshold recommended to identify obesity for Asians (32), hypertensive ICH patients, and patients 60 years and older, separately. The association of HOMA-IR with NLR and MLR was examined with Spearman analysis and multiple linear regression analysis.

All analyses were performed using the statistical package $\mathrm{R}$ (http://www.r-project.org) and EmpowerStats software (www. empowerstats.com, X\&Y Solutions, Inc., Boston, MA). A twotailed $p$ value $<0.05$ was considered to be statistically significant.

\section{RESULTS}

A total of 345 of 681 patients with ICH were finally enrolled in the study, after excluding those with isolated IVH, with secondary causes of hemorrhage, lacking insulin data or being treated with insulin, or with MRI unobtainable (Figure 1). Baseline characteristics of excluded patients because of missing insulin data or being treated with insulin are comparable with those who went into final analysis generally, except for the previous stroke/TIA occurrence, smoking status, and admission fasting glucose. When compared with patients excluded for lack of MRI data, the patients included in final analysis were less likely to experience severe neurological deficit, to have a history of $\mathrm{AF}, \mathrm{ICH}$, and smoking; had a higher BMI, TC, and LDL-C; and had smaller initial hematoma volume (Supplementary Table S1).

\section{Background Characteristics}

Among the 345 patients for final analysis, the mean age was 61.1 \pm 13.7 years, and $122(35.4 \%)$ patients were women. A total of 99 lesions were found in 54 patients $(15.7 \%$ in prevalence; range from 1 to 10 lesions per patient). The clinical and neuroimaging features of patients are reported in Table 1. The R-DWILs were more likely to occur in those who use antiplatelet drugs before ICH onset, those who presented ventricular hemorrhage, and those who had a higher HOMA-IR index or a higher total cSVD burden score. The median HOMA-IR was 2.11 (IQR, 1.33-4.04). Baseline characteristics of patients among different quartiles of HOMA-IR are summarized in Supplementary Table S2. Patients with higher HOMA-IR were younger, with a higher BMI and LDL-C level, and with higher score of NIHSS at admission, as well as being more likely to have a hypertension history and a higher initial blood pressure, with a higher frequency of diabetes, hypoglycemic treatments, and ventricle extension.

\section{Association Between HOMA-IR Index and Presence of R-DWILs}

The crude and adjusted association of HOMA-IR with R-DWILs are depicted in Figure 3A. The multivariable-adjusted ORs (95\% CIs) of R-DWIL occurrence across the quartiles of HOMA-IR increased with a higher HOMA-IR range. Patients with a HOMA-IR index of Q3 and Q4 were associated with higher risk of R-DWIL occurrence when compared with patients with a HOMA-IR index of Q1. The smoothing plot was also used to observe the relationship between the HOMA-IR index and RDWILs (Figure 4). We found that with the rise of HOMA-IR index, the risk of R-DWIL occurrence increased. The probability of R-DWILs reached a maximum when HOMA-IR closing to 10. However, as shown in the figure, the 95\% CIs of R-DWIL occurrence for nine patients with HOMA-IR $>10$ were too large and the threshold effect was not reflected properly. We performed a sensitivity analysis, and the multivariable-adjusted ORs of R-DWIL occurrence showed a similar association in nondiabetic patients (Figure 3B), non-obese patients (Figure 3C), hypertensive ICH patients (Figure 3D), and patients 60 years and older (Figure 3E).

\section{Association Between Insulin Resistance and Presence of R-DWILs in Non-Diabetic Patients}

Among the 280 non-diabetic patients, 86 (30.7\%) had insulin resistance based on the HOMA-IR index cutoff of 3.0. Compared 
TABLE 1 | Baseline characteristics of patients with and without R-DWIL.

\begin{tabular}{|c|c|c|c|c|}
\hline & $\begin{array}{l}\text { All patients } \\
\quad(n=345)\end{array}$ & $\begin{array}{l}\text { R-DWIL negative } \\
(\mathrm{n}=291)\end{array}$ & $\begin{array}{l}\text { R-DWIL positive } \\
(n=54)\end{array}$ & $p$ Value \\
\hline Age (years), mean (SD) & $61.1(13.7)$ & $61.0(13.9)$ & $61.8(12.9)$ & 0.679 \\
\hline Female, n (\%) & $122(35.4)$ & $101(34.7)$ & $21(38.9)$ & 0.555 \\
\hline BMl (kg/m2), mean (SD) & $24.0(4.0)$ & $24.0(4.0)$ & $24.1(4.1)$ & 0.622 \\
\hline NIHSS on admission, median (IQR) & $4(2,10)$ & $4(2,10)$ & $4(2,10)$ & 0.787 \\
\hline Systolic blood pressure (mmHg), mean (SD) & $161.3(25.5)$ & $160.4(24.6)$ & $165.7(30.0)$ & 0.413 \\
\hline Diastolic blood pressure (mmHg), mean (SD) & $91.0(17.2)$ & $90.4(16.1)$ & $94.2(22.4)$ & 0.601 \\
\hline History of hypertension, $\mathrm{n}(\%)$ & $265(76.8)$ & $219(75.3)$ & $46(85.2)$ & 0.112 \\
\hline History of diabetes mellitus, n (\%) & $65(18.8)$ & $52(17.9)$ & $13(24.1)$ & 0.284 \\
\hline History of atrial fibrillation, n (\%) & $10(2.9)$ & $7(2.4)$ & $3(5.6)$ & 0.409 \\
\hline History of $\mathrm{ICH}, \mathrm{n}(\%)$ & $24(7.0)$ & $22(7.6)$ & $2(3.7)$ & 0.464 \\
\hline History of $\mathrm{Cl} / \mathrm{TIA}, \mathrm{n}(\%)$ & $38(11.0)$ & $28(9.6)$ & $10(18.5)$ & 0.055 \\
\hline Smoking status, n (\%) & & & & 0.556 \\
\hline Smoker or ex-smoker & $101(29.3)$ & $87(29.9)$ & $14(25.9)$ & \\
\hline Non-smoker & $244(70.7)$ & $204(70.1)$ & $40(74.1)$ & \\
\hline Drinking status, n (\%) & & & & 0.322 \\
\hline Drinker or ex-drinker & $116(33.6)$ & $101(34.7)$ & $15(27.8)$ & \\
\hline Non-drinker & $229(66.4)$ & $190(65.3)$ & $39(72.2)$ & \\
\hline \multicolumn{5}{|l|}{ Medicine use before admission, $\mathrm{n}(\%)$} \\
\hline Antiplatelet drug & $31(9.0)$ & $19(6.5)$ & $12(22.2)$ & 0.001 \\
\hline Anticoagulant drug & $7(2.0)$ & $6(2.1)$ & $1(1.9)$ & 0.92 \\
\hline Antihypertensive drug & $155(44.9)$ & $126(43.3)$ & $29(53.7)$ & 0.158 \\
\hline Hypoglycemic drug & $47(13.6)$ & $38(13.1)$ & $9(16.7)$ & 0.478 \\
\hline Statin & $26(7.5)$ & $20(6.9)$ & $6(11.1)$ & 0.422 \\
\hline TC (mmol/L), mean (SD) & $4.8(1.1)$ & $4.7(1.2)$ & $4.9(1.1)$ & 0.473 \\
\hline LDL-C (mmol/L), mean (SD) & $2.5(0.8)$ & $2.5(0.8)$ & $2.5(0.7)$ & 0.784 \\
\hline eGFR $\left(\mathrm{ml} / \mathrm{min} / 1.73 \mathrm{~m}^{2}\right)$, median (IQR) & $99.9(91.4,109.8)$ & $99.9(91.1,110.0)$ & $100.8(92.7,109.5)$ & 0.664 \\
\hline HOMA-IR, median (IQR) & $2.1(1.3,4.0)$ & $2.0(1.3,3.8)$ & $3.0(2.0,4.6)$ & 0.002 \\
\hline Hematoma volume (ml), median (IQR) & $7.8(3.0,17.0)$ & $7.8(3.1,16.6)$ & $7.7(2.7,20.2)$ & 0.789 \\
\hline Hematoma location, n (\%) & & & & 0.361 \\
\hline Lobar & $68(19.7)$ & $54(18.6)$ & $14(25.9)$ & \\
\hline Deep & $214(62.0)$ & $181(62.2)$ & $33(61.1)$ & \\
\hline Infratentorial & $44(12.7)$ & $41(14.1)$ & $3(5.6)$ & \\
\hline Mixed & $19(5.5)$ & $15(5.2)$ & $4(7.4)$ & \\
\hline Ventricle extension, n (\%) & $102(29.6)$ & $78(26.8)$ & $24(44.4)$ & 0.009 \\
\hline Subarachnoid extension, n (\%) & $31(9.0)$ & $27(9.3)$ & $4(7.4)$ & 0.855 \\
\hline Total cSVD burden, median (IQR) & $2(1,3.8)$ & $2(1,3)$ & $3(2,4)$ & $<0.001$ \\
\hline Time to MRI (days), median (IQR) & $6(5,8)$ & $6(5,8)$ & $6(5,8)$ & 0.747 \\
\hline Presumed etiology of $\mathrm{ICH}, \mathrm{n}(\%)$ & & & & 0.268 \\
\hline $\mathrm{HA}$ & $243(70.4)$ & $200(68.7)$ & $43(79.6)$ & \\
\hline CAA & $40(11.6)$ & $36(12.4)$ & $4(7.4)$ & \\
\hline Anticoagulation or undetermined cause & $62(18.0)$ & 55 (18.9) & $7(13.0)$ & \\
\hline
\end{tabular}

R-DWIL indicates remote diffusion-weighted imaging lesion; SD, standard deviation; BMI, body mass index; IQR, interquartile range; NIHSS, national institute of health stroke scale; ICH, intracerebral hemorrhage; Cl, cerebral infarction; TIA, transient ischemic attack; TC, total cholesterol; LDL-C, low-density lipoprotein-cholesterol; eGFR, estimated glomerular fltration rate;

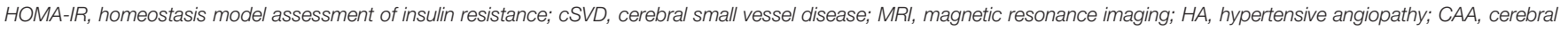
amyloid angiopathy.

with patients without insulin resistance, those with insulin resistance were more likely to have R-DWILs (OR, 2.84; 95\% CI, 1.20-6.74; $p=0.018$; Figure 3B) after adjustment with the potential confounders.

\section{Association of Insulin Resistance With NLR and MLR}

After excluding patients with active infections before or during hospitalization, or with severe hepatic or renal diseases, 277 patients remained for analyses. NLR and MLR were both correlated with HOMA-IR. Multiple linear regression analysis was then applied and identified independent association of insulin resistance with NLR and MLR after adjusting for age, history of hypertension, statin use, and smoking (Table 2).

\section{DISCUSSION}

In this study, we found that insulin resistance evaluated by HOMA-IR index was associated with R-DWIL occurrence in patients with primary $\mathrm{ICH}$ within 14 days after symptom onset. The association was consistent in patients without diabetes or obesity. Moreover, the significant relationship was also shown in patients with etiology of hypertensive angiopathy or in elderly patients.

Insulin resistance is a metabolic disorder resulting in wideranging effects on many organs and insulin-regulated pathways. It is characterized by a reduced action of insulin despite increased insulin concentrations (33). Emerging works indicated that insulin resistance prevents recovery from 


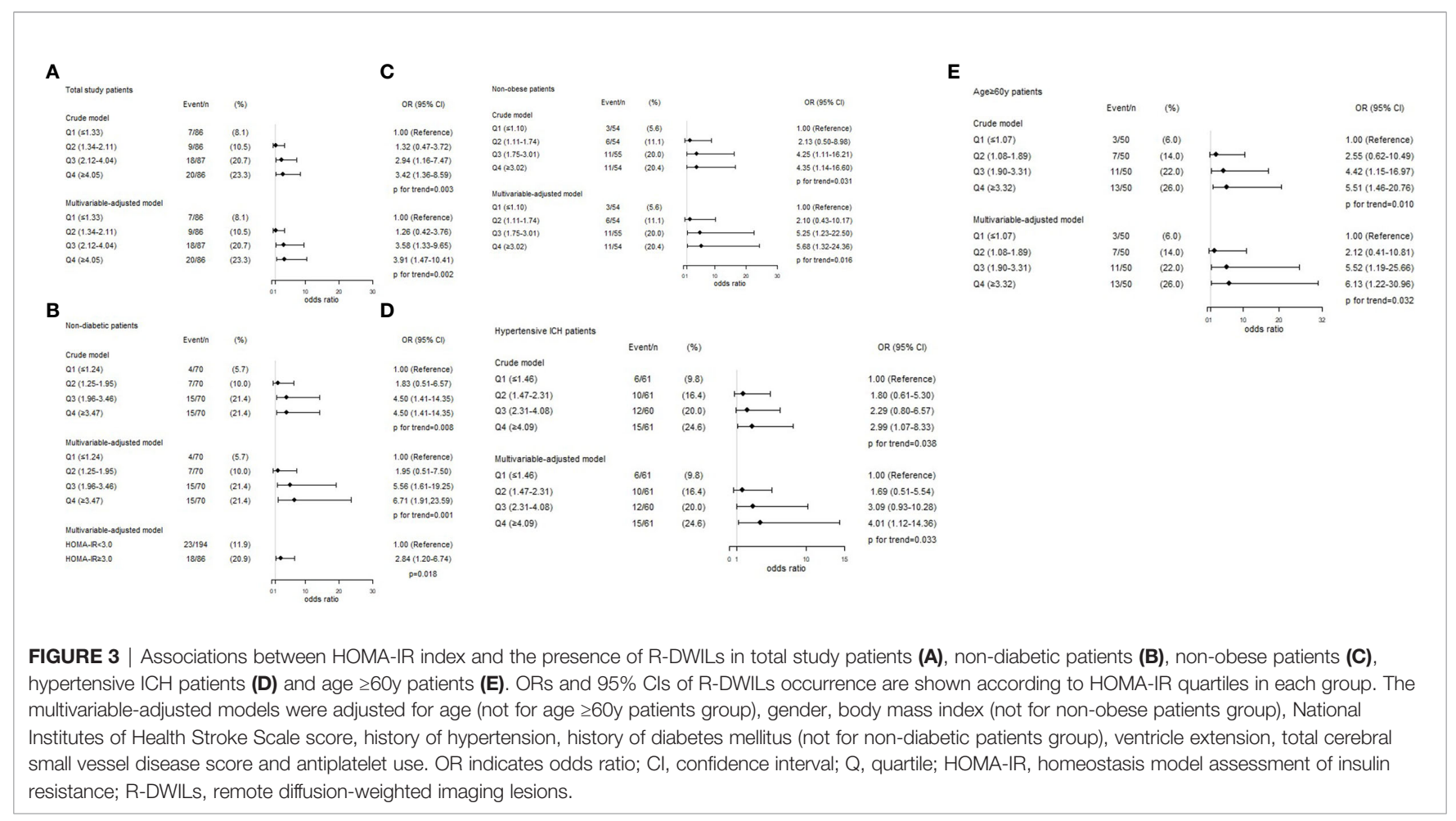

ischemic injury and subsequently worsened the functional outcomes after acute ischemic stroke (11-14). Insulin resistance also had adverse impact on non-diabetic patients with ischemic stroke or TIA $(11,12)$. However, existing evidence about the impacts of insulin resistance on ICH patients is scarce and controversial. In a large populationbased Rotterdam study with 5,234 non-diabetic elderly

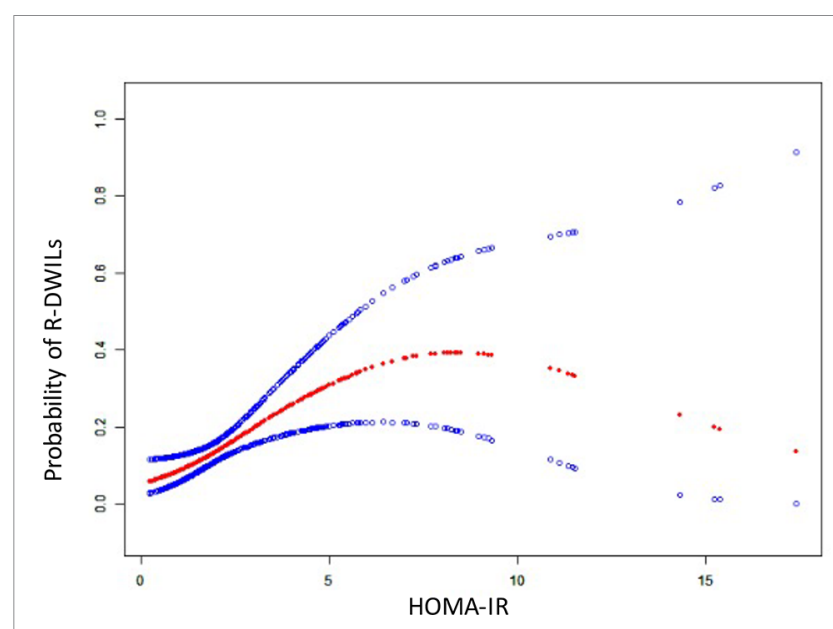

FIGURE 4 | The relationship between HOMA-IR and the probability of R-DWILs. A nonlinear relationship between HOMA-IR and the probability of R-DWILs was observed after adjusted for age, gender, body mass index, National Institutes of Health Stroke Scale score, history of hypertension, history of diabetes mellitus, ventricle extension, total cerebral small vessel disease score and antiplatelet use. The probabilities and 95\% Cls of R-DWILs occurrence are shown in red dotted line and blue dotted lines respectively. enrolled, insulin resistance was not associated with the risk of ICH (34). The REasons for Geographic And Racial Differences in Stroke (REGARDS) study reported a nonsignificant yet discordant tendency of ICH risk in patients at higher levels of insulin resistance, with a downward trend in Whites and an upward trend in Blacks (35).

Our present work uncovered that insulin resistance was common not only in the overall ICH patients but also in nondiabetic and non-obese ICH patients. Because the HOMA-IR index is influenced by race, age, and baseline diseases, there is no universally accepted threshold to define insulin resistance with HOMA-IR. In this context, many investigators identified insulin resistance by the top quartile of HOMA-IR index in non-diabetic $(11,15,34)$, others applied varied cutoffs (range, from 2.5 to 3.0) in discrepant populations among studies $(22,36)$. Interestingly, we found that patients in the fourth quartile of HOMA-IR along with a proportion of patients in the third quartile were indeed experiencing impaired insulin sensitivity, with reference of cutoffs mentioned above.

We previously confirmed that fasting blood glucose on admission was associated with the development of R-DWILs in ICH patients, whereas diabetes history showed no relation to it (3). It may partially be explained with the fact that glucose metabolic abnormality is common even among individuals without diagnosed diabetes (4). On the basis of this primary ICH cohort, we evaluated the association of insulin resistance with the presence of R-DWILs and reached a statistical correlation. Because diabetes and obesity are two metabolic syndrome components that closely correlated with insulin 
TABLE 2 | Association of HOMA-IR index with NLR and MLR.

HOMA-IR

\begin{tabular}{lcccc} 
& \multicolumn{2}{c}{ Spearman analysis } & & \multicolumn{2}{c}{ Multiple linear regression analysis } \\
\cline { 2 - 3 } & $\boldsymbol{r}$ & $\boldsymbol{p}$ Value & Standardized coefficient & $\boldsymbol{p}$ Value \\
\hline NLR & 0.167 & 0.005 & 0.147 & 0.016 \\
MLR & 0.121 & 0.044 & 0.174 & 0.004 \\
\hline
\end{tabular}

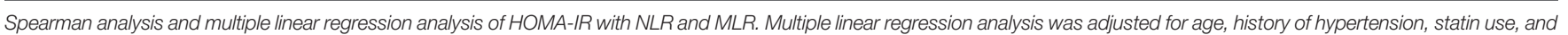
smoking. HOMA-IR indicates homeostasis model assessment of insulin resistance; NLR, neutrophil to lymphocyte ratio; MLR, monocyte to lymphocyte ratio.

resistance, we also conducted analysis among non-diabetic and non-obese patients and yielded out similar results.

It is recognized that chronic low-grade inflammation is involved in the pathogenesis of insulin resistance, and in turn insulin resistance can reflect existing systemic inflammatory status (37). In our study, HOMA-IR was positively associated with systemic inflammatory immune indices NLR and MLR, meaning patients with severe insulin resistance suffering increased systemic inflammation. In the setting of $\mathrm{ICH}$, already destroyed $\mathrm{BBB}$ allows invasion of peripheral immune cells and proinflammatory cytokines to the brain. Increased neuroinflammation results in further disruption of $\mathrm{BBB}(16,17)$. Patients with insulin resistance were supposed to suffer from more significant $\mathrm{BBB}$ integrity deterioration. Moreover, dysregulated insulin signaling in brain activates NFKB transcription factors aggravating neuroinflammation (18). Inflammatory injury with abnormal tissue environment and remote extension of hematoma constituents through perivascular and perineural space were speculated as potential mechanisms of R-DWILs $(3,25)$.

The endothelial dysfunction also contributes to R-DWILs formation in ICH patients with insulin resistance. Because of the insulin receptor expressed in endothelium, insulin acts on vasculature as well, in addition to classical insulin target tissues like liver, skeletal muscle, and white adipose tissue (33). Under normal conditions, insulin acts on the insulin receptor of endothelial cells, leading downstream to phosphatidylinositol 3-kinase (PI3K)-Akt activation. Akt phosphorylates endothelial nitric oxide (NO) synthase to catalyze the production of NO from L-arginine, thereby resulting in vessel relaxation (38). However, under insulin resistance status, this pathway is impaired, whereas the mitogen-activated protein kinase (MAPK) pathway is preponderant and vasoconstriction is stimulated. What is worse, the activated endothelia express adhesion proteins, such as vascular cell adhesion molecule-1 and E-selectin, attract leukocytes and activate platelets, altogether leading to elevated permeability of the endothelia and potentially the development of local atherosclerosis (33). R-DWILs may be in situ small vessel occlusion implying an ischemia-prone state after ICH and may be partially due to this insulin resistance-related vessel injury (25).

Adequate cerebral perfusion is essential for brain function, whereas improper blood supply could lead to ischemia and brain damage within minutes. Cerebral autoregulation is one of the well-developed mechanisms in the brain to prevent from fluctuating of cerebral blood flow (39). Recent study has suggested that in acute ICH patients, with elevating intracranial pressure, the dynamic cerebral autoregulation was globally impaired within about 2 weeks (40). Among several elements contributing to the cerebral autoregulation, myogenic response, the ability of smooth muscle cells reacting to blood pressure changes, is the most prominent one (39). Studies in rats demonstrated that insulin resistance provoked an increase in cerebrovascular myogenic tone and a decrease in lumen diameter $(41,42)$, which could partly explain the increased risk of R-DWILs in patients with more severe insulin resistance after ICH. Besides, distal arteries possess a substantially higher myogenic activity than proximal arteries (39) and could be more vulnerable to insulin resistanceinduced damage. Consistent with it, R-DWILs are mainly located in cortical or subcortical regions and are mostly small, dot-like.

HA and CAA are the two main etiologies for primary $\mathrm{ICH}$. A few studies showed that R-DWILs were more commonly seen in patients with CAA-related $\mathrm{ICH}$ than in patients with HA-related ICH $(43,44)$. Although in other studies, similar occurrence of R-DWILs were observed in these two etiologies of $\mathrm{ICH}$ (45). Insulin resistance is a systemic metabolic abnormality, which has been proven to be related to other metabolic disorders closely, including hypertension (36). Insulin resistance is also associated with an increased level of amyloid- $\beta 42(\mathrm{~A} \beta 42)$ in Alzheimer disease (AD) brains (16). Meanwhile, $A \beta$ deposition in arteries is regarded as the cause of CAA. Because insulin resistance is correlated with both hypertension and accumulation of $\mathrm{A} \beta$, we wonder if $\mathrm{ICH}$ patients with different etiologies may possess varied insulin resistance severity. We tried to explore the associations of RDWIL occurrence with insulin resistance under different etiologies of ICH but yielded out null results.

In recent studies, piles of evidence revealed that the occurrence of R-DWILs was correlated with the total burden of cSVD. In our former study, we found that R-DWILs were more likely to present in patients with higher total cSVD burden (26). In a previous study in elderly, a nondiabetic, healthy population showed that insulin resistance was a major risk factor of cSVD burden (46). Thus, it is necessary to identify if cSVD burden would play a role in the association between insulin resistance and R-DWILs. Our present work found a meaningful association between cSVD burden and R-DWIL occurrence. However, we did not find a statistical relationship between HOMA-IR and cSVD burden, which may be partially 
because of the difference in study cohort and CSVD burden scoring methodology. Moreover, after being adjusted with potential confounders, including total cSVD score, the independent association between insulin resistance and $\mathrm{R}$ DWILs still existed. It gave a reason to assume that insulin resistance was correlated with R-DWIL occurrence, independent of cSVD burden.

Besides, the ventricle extension of hemorrhage may play a role in the association between insulin resistance and R-DWILs. Elevated intraventricular concentrations of inflammatory markers were observed in ICH patients accompanied with IVH (47). Blood constitutes, inflammatory cells, and proinflammatory factors could extend through cerebrospinal fluid, resulting in brain injury in remote areas. In patients with severe insulin resistance in our cohort, a higher frequency of IVH was detected. The underlying mechanism is unclear, and further explorations are needed. However, the global neuroinflammation and deteriorated $\mathrm{BBB}$ in the insulin resistance setting was supposed to be coordinated with IVH regarding to R-DWIL occurrence.

This study has several limitations. Because of missing insulin data or being treated with insulin or lacking MRI data, 271 patients were ruled out from our study. The baseline characteristics between the patients included in and excluded from the analysis were not well balanced, especially those excluded for unavailable MRI data were more likely to suffer from fatal hemorrhage and experience severe clinical symptoms. This may lead to selection bias, whereas generalizability of the findings should be further validated in cohort, including severe cases. Another possible criticism of our study is that we collected blood sample for insulin sensitivity measurement too soon after ICH onset, which may not represent actual steady-state values. The cerebrovascular event itself may temporarily reduce insulin sensitivity, and then the insulin resistance prevalence may be overestimated (48). Although HOMA-IR correlated strongly with insulin resistance estimated by the gold standard of euglycemic-hyperinsulinemic clamp and is the most widely accepted surrogate measure of insulin resistance, there should be a cautious interpretation of the current finding.

\section{CONCLUSIONS}

Our study demonstrated that insulin resistance was associated with the occurrence of R-DWILs in ICH patients within 14 days after symptom onset. This result shed light on insulin resistance as a potential metabolic factor involved in brain injury after ICH.

\section{REFERENCES}

1. Kim JY, Bae HJ. Spontaneous Intracerebral Hemorrhage: Management. J Stroke (2017) 19(1):28-39. doi: 10.5853/jos.2016.01935

2. Hemphill JC3rd, Greenberg SM, Anderson CS, Becker K, Bendok BR, Cushman M, et al. Guidelines for the Management of Spontaneous Intracerebral Hemorrhage: A Guideline for Healthcare Professionals From the American Heart Association/American Stroke Association. Stroke (2015) 46(7):2032-60. doi: 10.1161/STR.0000000000000069

\section{DATA AVAILABILITY STATEMENT}

The raw data supporting the conclusions of this article will be made available by the authors, without undue reservation.

\section{ETHICS STATEMENT}

The studies involving human participants were reviewed and approved by the institutional Human Research Ethics Committee of the Second Affiliated Hospital of Zhejiang University. Written informed consent for participation was not required for this study in accordance with the national legislation and the institutional requirements.

\section{AUTHOR CONTRIBUTIONS}

X-HY brought up the main idea, developed the protocol, collected and analyzed data, and wrote the manuscript. J-LZ collected the data, searched for literatures, and provided helpful input on the theme. Y-JJ helped analyzing data, found some useful papers, and offered some helpful suggestions. DS collected part of the data and searched for useful papers. J-WL, X-DH, and J-WZ helped to read the images and collected part of the data. L-HJ collected part of the data. FG and L-ST helped developing the protocol, supervised and offered guidance to all the authors, revised the manuscript, and polished the language. All authors contributed to the article and approved the submitted version.

\section{FUNDING}

This work was supported by grants from the National Natural Science Foundation of China (NSFC) (81971155) to L-ST, the National Natural Science Foundation of China (NSFC) (81471168) to FG, and Science and Technology Action Plan for Major Diseases Prevention and Control in China to FG (2017ZX-01S-006S3).

\section{SUPPLEMENTARY MATERIAL}

The Supplementary Material for this article can be found online at: https://www.frontiersin.org/articles/10.3389/fimmu.2021. 719462/full\#supplementary-material

3. Ye XH, Gao T, Xu XH, Cai JS, Li JW, Liu KM, et al. Factors Associated With Remote Diffusion-Weighted Imaging Lesions in Spontaneous Intracerebral Hemorrhage. Front Neurol (2018) 9:209. doi: 10.3389/fneur.2018.00209

4. Ye XH, Cai XL, Nie DL, Chen YJ, Li JW, Xu XH, et al. Stress-Induced Hyperglycemia and Remote Diffusion-Weighted Imaging Lesions in Primary Intracerebral Hemorrhage. Neurocrit Care (2020) 32(2):427-36. doi: 10.1007/ s12028-019-00747-y

5. Garg RK, Liebling SM, Maas MB, Nemeth AJ, Russell EJ, Naidech AM. Blood Pressure Reduction, Decreased Diffusion on MRI, and Outcomes After 
Intracerebral Hemorrhage. Stroke (2012) 43(1):67-71. doi: 10.1161/ STROKEAHA.111.629493

6. Kang DW, Han MK, Kim HJ, Yun SC, Jeon SB, Bae HJ, et al. New Ischemic Lesions Coexisting With Acute Intracerebral Hemorrhage. Neurology (2012) 79(9):848-55. doi: 10.1212/WNL.0b013e3182648a79

7. Garg RK, Khan J, Dawe RJ, Conners J, John S, Prabhakaran S, et al. The Influence of Diffusion Weighted Imaging Lesions on Outcomes in Patients With Acute Spontaneous Intracerebral Hemorrhage. Neurocrit Care (2020) 33(2):552-64. doi: 10.1007/s12028-020-00933-3

8. Kidwell CS, Rosand J, Norato G, Dixon S, Worrall BB, James ML, et al. Ischemic Lesions, Blood Pressure Dysregulation, and Poor Outcomes in Intracerebral Hemorrhage. Neurology (2017) 88(8):782-8. doi: 10.1212/ WNL.0000000000003630

9. Murthy SB, Cho SM, Gupta A, Shoamanesh A, Navi BB, Avadhani R, et al. A Pooled Analysis of Diffusion-Weighted Imaging Lesions in Patients With Acute Intracerebral Hemorrhage. JAMA Neurol (2020) 77(11):1390-7. doi: $10.1001 /$ jamaneurol.2020.2349

10. Kernan WN, Inzucchi SE, Viscoli CM, Brass LM, Bravata DM, Horwitz RI. Insulin Resistance and Risk for Stroke. Neurology (2002) 59(6):809-15. doi: $10.1212 / \mathrm{wnl} .59 .6 .809$

11. Jing J, Pan Y, Zhao X, Zheng H, Jia Q, Mi D, et al. Insulin Resistance and Prognosis of Nondiabetic Patients With Ischemic Stroke: The ACROSS-China Study (Abnormal Glucose Regulation in Patients With Acute Stroke Across China). Stroke (2017) 48(4):887-93. doi: 10.1161/STROKEAHA.116.015613

12. Pan Y, Jing J, Chen W, Zheng H, Jia Q, Mi D, et al. Post-Glucose Load Measures of Insulin Resistance and Prognosis of Nondiabetic Patients With Ischemic Stroke. J Am Heart Assoc (2017) 6(1):e004990. doi: 10.1161/ JAHA.116.004990

13. Ago T, Matsuo R, Hata J, Wakisaka Y, Kuroda J, Kitazono T, et al. Insulin Resistance and Clinical Outcomes After Acute Ischemic Stroke. Neurology (2018) 90(17):e1470-e7. doi: 10.1212/WNL.0000000000005358

14. Bas DF, Ozdemir AO, Colak E, Kebapci N. Higher Insulin Resistance Level is Associated With Worse Clinical Response in Acute Ischemic Stroke Patients Treated With Intravenous Thrombolysis. Transl Stroke Res (2016) 7(3):16771. doi: 10.1007/s12975-016-0453-y

15. Rundek T, Gardener H, Xu Q, Goldberg RB, Wright CB, Boden-Albala B, et al. Insulin Resistance and Risk of Ischemic Stroke Among Nondiabetic Individuals From the Northern Manhattan Study. Arch Neurol (2010) 67 (10):1195-200. doi: 10.1001/archneurol.2010.235

16. de la Monte SM. Insulin Resistance and Neurodegeneration: Progress Towards the Development of New Therapeutics for Alzheimer's Disease. Drugs (2017) 77(1):47-65. doi: 10.1007/s40265-016-0674-0

17. Van Dyken P, Lacoste B. Impact of Metabolic Syndrome on Neuroinflammation and the Blood-Brain Barrier. Front Neurosci (2018) 12:930. doi: $10.3389 /$ fnins. 2018.00930

18. Akhtar A, Sah SP. Insulin Signaling Pathway and Related Molecules: Role in Neurodegeneration and Alzheimer's Disease. Neurochem Int (2020) 135:104707. doi: 10.1016/j.neuint.2020.104707

19. Knudsen KA, Rosand J, Karluk D, Greenberg SM. Clinical Diagnosis of Cerebral Amyloid Angiopathy: Validation of the Boston Criteria. Neurology (2001) 56(4):537-9. doi: 10.1212/wnl.56.4.537

20. Teo BW, Xu H, Wang D, Li J, Sinha AK, Shuter B, et al. GFR Estimating Equations in a Multiethnic Asian Population. Am J Kidney Dis Off J Natl Kidney Foundation (2011) 58(1):56-63. doi: 10.1053/j.ajkd.2011.02.393

21. Matthews DR, Hosker JP, Rudenski AS, Naylor BA, Treacher DF, Turner RC. Homeostasis Model Assessment: Insulin Resistance and Beta-Cell Function From Fasting Plasma Glucose and Insulin Concentrations in Man. Diabetologia (1985) 28(7):412-9. doi: 10.1007/BF00280883

22. Kernan WN, Viscoli CM, Furie KL, Young LH, Inzucchi SE, Gorman M, et al. Pioglitazone After Ischemic Stroke or Transient Ischemic Attack. New Engl J Med (2016) 374(14):1321-31. doi: 10.1056/NEJMoa1506930

23. Wang J, Zhu QW, Cheng XY, Liu JY, Zhang LL, Tao YM, et al. Assessment Efficacy of Neutrophil-Lymphocyte Ratio and Monocyte-Lymphocyte Ratio in Preeclampsia. J Reprod Immunol (2019) 132:29-34. doi: 10.1016/ j.jri.2019.02.001

24. Kothari RU, Brott T, Broderick JP, Barsan WG, Sauerbeck LR, Zuccarello M, et al. The Abcs of Measuring Intracerebral Hemorrhage Volumes. Stroke (1996) 27(8):1304-5. doi: 10.1161/01.str.27.8.1304
25. Xu XH, Gao T, Zhang WJ, Tong LS, Gao F. Remote Diffusion-Weighted Imaging Lesions in Intracerebral Hemorrhage: Characteristics, Mechanisms, Outcomes, and Therapeutic Implications. Front Neurol (2017) 8:678. doi: 10.3389/fneur.2017.00678

26. Xu XH, Ye XH, Li JW, Cai JS, Gao T, Zhang WJ, et al. Association Between Remote Diffusion-Weighted Imaging Lesions and Cerebral Small Vessel Disease in Primary Intracerebral Hemorrhage. Eur J Neurol (2019) 26 (7):961-8. doi: 10.1111/ene.13915

27. Greenberg SM, Vernooij MW, Cordonnier C, Viswanathan A, Al-Shahi Salman R, Warach S, et al. Cerebral Microbleeds: A Guide to Detection and Interpretation. Lancet Neurol (2009) 8(2):165-74. doi: 10.1016/S1474-4422 (09)70013-4

28. Fazekas F, Barkhof F, Wahlund LO, Pantoni L, Erkinjuntti T, Scheltens P, et al. CT and MRI Rating of White Matter Lesions. Cerebrovascular Dis (2002) 13(Suppl 2):31-6. doi: 10.1159/000049147

29. Maclullich AM, Wardlaw JM, Ferguson KJ, Starr JM, Seckl JR, Deary IJ. Enlarged Perivascular Spaces Are Associated With Cognitive Function in Healthy Elderly Men. J Neurology Neurosurgery Psychiatry (2004) 75 (11):1519-23. doi: 10.1136/jnnp.2003.030858

30. Hansen TP, Cain J, Thomas O, Jackson A. Dilated Perivascular Spaces in the Basal Ganglia are a Biomarker of Small-Vessel Disease in a Very Elderly Population With Dementia. AJNR Am J Neuroradiol (2015) 36(5):893-8. doi: 10.3174/ajnr.A4237

31. Lau KK, Li L, Schulz U, Simoni M, Chan KH, Ho SL, et al. Total Small Vessel Disease Score and Risk of Recurrent Stroke: Validation in 2 Large Cohorts. Neurology (2017) 88(24):2260-7. doi: 10.1212/WNL.0000000000004042

32. Wang T, Lu J, Shi L, Chen G, Xu M, Xu Y, et al. Association of Insulin Resistance and Beta-Cell Dysfunction With Incident Diabetes Among Adults in China: A Nationwide, Population-Based, Prospective Cohort Study. Lancet Diabetes Endocrinol (2020) 8(2):115-24. doi: 10.1016/S2213-8587(19)30425-5

33. Artunc F, Schleicher E, Weigert C, Fritsche A, Stefan N, Haring HU. The Impact of Insulin Resistance on the Kidney and Vasculature. Nat Rev Nephrol (2016) 12(12):721-37. doi: 10.1038/nrneph.2016.145

34. Wieberdink RG, Koudstaal PJ, Hofman A, Witteman JC, Breteler MM, Ikram MA. Insulin Resistance and the Risk of Stroke and Stroke Subtypes in the Nondiabetic Elderly. Am J Epidemiol (2012) 176(8):699-707. doi: 10.1093/aje/ kws149

35. Howard G, Wagenknecht LE, Kernan WN, Cushman M, Thacker EL, Judd SE, et al. Racial Differences in the Association of Insulin Resistance With Stroke Risk: The Reasons for Geographic and Racial Differences in Stroke (REGARDS) Study. Stroke (2014) 45(8):2257-62. doi: 10.1161/ STROKEAHA.114.005306

36. Lee JE, Shin DW, Yun JM, Kim SH, Nam YS, Cho B, et al. Insulin Resistance is a Risk Factor for Silent Lacunar Infarction. Stroke (2016) 47(12):2938-44. doi: 10.1161/STROKEAHA.116.014097

37. Glass CK, Olefsky JM. Inflammation and Lipid Signaling in the Etiology of Insulin Resistance. Cell Metab (2012) 15(5):635-45. doi: 10.1016/ j.cmet.2012.04.001

38. Tokarz VL, MacDonald PE, Klip A. The Cell Biology of Systemic Insulin Function. J Cell Biol (2018) 217(7):2273-89. doi: 10.1083/jcb.201802095

39. Coucha M, Abdelsaid M, Ward R, Abdul Y, Ergul A. Impact of Metabolic Diseases on Cerebral Circulation: Structural and Functional Consequences. Compr Physiol (2018) 8(2):773-99. doi: 10.1002/cphy.c170019

40. Ma H, Guo ZN, Liu J, Xing Y, Zhao R, Yang Y. Temporal Course of Dynamic Cerebral Autoregulation in Patients With Intracerebral Hemorrhage. Stroke (2016) 47(3):674-81. doi: 10.1161/STROKEAHA.115.011453

41. Butcher JT, Goodwill AG, Stanley SC, Frisbee JC. Differential Impact of Dilator Stimuli on Increased Myogenic Activation of Cerebral and Skeletal Muscle Resistance Arterioles in Obese Zucker Rats. Microcirculation (2013) 20(7):579-89. doi: 10.1111/micc.12056

42. Phillips SA, Sylvester FA, Frisbee JC. Oxidant Stress and Constrictor Reactivity Impair Cerebral Artery Dilation in Obese Zucker Rats. Am J Physiol Regul Integr Comp Physiol (2005) 288(2):R522-30. doi: 10.1152/ ajpregu.00655.2004

43. Gregoire SM, Charidimou A, Gadapa N, Dolan E, Antoun N, Peeters A, et al. Acute Ischaemic Brain Lesions in Intracerebral Haemorrhage: Multicentre Cross-Sectional Magnetic Resonance Imaging Study. Brain J Neurol (2011) 134(Pt 8):2376-86. doi: 10.1093/brain/awr172 
44. Wu B, Yao X, Lei C, Liu M, Selim MH. Enlarged Perivascular Spaces and Small Diffusion-Weighted Lesions in Intracerebral Hemorrhage. Neurology (2015) 85(23):2045-52. doi: 10.1212/WNL.0000000000002169

45. Auriel E, Gurol ME, Ayres A, Dumas AP, Schwab KM, Vashkevich A, et al. Characteristic Distributions of Intracerebral Hemorrhage-Associated DiffusionWeighted Lesions. Neurology (2012) 79(24):2335-41. doi: 10.1212/ WNL.0b013e318278b66f

46. Yang X, Zhang S, Dong Z, Zi Y, Luo Y, Jin Z, et al. Insulin Resistance is a Risk Factor for Overall Cerebral Small Vessel Disease Burden in Old Nondiabetic Healthy Adult Population. Front Aging Neurosci (2019) 11:127. doi: 10.3389/fnagi.2019.00127

47. Ziai W, Parry-Jones A, Nekoovaght-Tak S, Mould A, Thompson C, Murthy S, et al. Time Trends of Inflammatory Markers in Cerebrospinal Fluid of Patients With Spontaneous Intracerebral and Intraventricular Hemorrhage (S39.002). Neurology (2015) 84(14 Supplement):S39.002. doi: 10.1002/14356007.b08_001.pub2

48. Huff TA, Lebovitz HE, Heyman A, Davis L. Serial Changes in Glucose Utilization and Insulin and Growth Hormone Secretion in Acute Cerebrovascular Disease. Stroke (1972) 3(5):543-52. doi: 10.1161/01.str.3.5.543
Conflict of Interest: The authors declare that the research was conducted in the absence of any commercial or financial relationships that could be construed as a potential conflict of interest.

Publisher's Note: All claims expressed in this article are solely those of the authors and do not necessarily represent those of their affiliated organizations, or those of the publisher, the editors and the reviewers. Any product that may be evaluated in this article, or claim that may be made by its manufacturer, is not guaranteed or endorsed by the publisher.

Copyright $\odot 2021$ Ye, Zhang, Jin, Shen, Hao, Li, Zhong, Jin, Tong and Gao. This is an open-access article distributed under the terms of the Creative Commons Attribution License (CC BY). The use, distribution or reproduction in other forums is permitted, provided the original author(s) and the copyright owner(s) are credited and that the original publication in this journal is cited, in accordance with accepted academic practice. No use, distribution or reproduction is permitted which does not comply with these terms. 